\title{
Jumping into a Healthier Future: Trampolining for Increasing Physical Activity in Children
}

\author{
Isabelle Schöffl ${ }^{1,2^{*}}$ (D, Benedikt Ehrlich ${ }^{3}$, Kathrin Rottermann ${ }^{1}$, Annika Weigelt', Sven Dittrich ${ }^{1}$ and Volker Schöffl ${ }^{2,3,4}$
}

\begin{abstract}
Objectives: Physical activity in children and adolescents has positive effects on cardiopulmonary function in this age group as well as later in life. As poor cardiopulmonary function is associated with higher mortality and morbidity, increasing physical activity especially in children needs to become a priority. Trampoline jumping is widely appreciated in children. The objective was to investigate its use as a possible training modality.
\end{abstract}

Methods: Fifteen healthy children (10 boys and 5 girls) with a mean age of 8.8 years undertook one outdoor incremental running test using a mobile cardiopulmonary exercise testing unit. After a rest period of at least 2 weeks, a trampoline test using the mobile unit was realized by all participants consisting of a 5-min interval of moderate-intensity jumping and two high-intensity intervals with vigorous jumping for 2 min, interspersed with 1min rests.

Results: During the interval of moderate intensity, the children achieved $\mathrm{VO}_{2}$-values slightly higher than the first ventilatory threshold (VT1) and during the high-intensity interval comparable to the second ventilatory threshold (VT2) of the outdoor incremental running test. They were able to maintain these values for the duration of the respective intervals. The maximum values recorded during the trampoline test were significantly higher than during the outdoor incremental running test.

Conclusion: Trampoline jumping is an adequate tool for implementing high-intensity interval training as well as moderate-intensity continuous training in children. As it is a readily available training device and is greatly enjoyed in this age group, it could be implemented in exercise interventions.

Keywords: Cardiopulmonary function, Exercise testing, Rehabilitation, Training modalities, Pediatric cardiology, Training, Children, Pediatrics, Physical activity

\section{Key Points}

- Trampolining is a widely used physical activity in children and adolescents.

- As the proportion of children achieving the required 60 min of physical daily activity has declined

\footnotetext{
* Correspondence: isabelle.schoeffl@me.com

'Department of Pediatric Cardiology, University Hospital

Erlangen-Nuremberg, 91054 Erlangen, Germany

${ }^{2}$ School of Clinical and Applied Sciences, Leeds Beckett University, Leeds,

Great Britain

Full list of author information is available at the end of the article
}

dramatically over the last years, motivating children and developing adequate training methods are crucial.

- High-intensity interval training (HIIT) provides a more time-efficient approach with a higher fun aspect, underlining the need for developing ageappropriate training methods with a focus on HIIT.

- Trampolining achieves intensities of oxygen uptake $\left(\mathrm{VO}_{2}\right)$ and heart rate above the second ventilatory threshold and is therefore suitable for HIIT.

\section{Springer Open}

(c) The Author(s). 2021 Open Access This article is licensed under a Creative Commons Attribution 4.0 International License, which permits use, sharing, adaptation, distribution and reproduction in any medium or format, as long as you give appropriate credit to the original author(s) and the source, provide a link to the Creative Commons licence, and indicate if changes were made. The images or other third party material in this article are included in the article's Creative Commons licence, unless indicated otherwise in a credit line to the material. If material is not included in the article's Creative Commons licence and your intended use is not permitted by statutory regulation or exceeds the permitted use, you will need to obtain permission directly from the copyright holder. To view a copy of this licence, visit http://creativecommons.org/licenses/by/4.0/. 
- This is the first study using cardiopulmonary exercise equipment for evaluating trampolining for its use for HIIT in children.

\section{Introduction}

Cardiorespiratory fitness (CRF) is an objective, reproducible, and physiological measure [1] that can be recorded by using maximal graded cardiorespiratory exercise testing and determining peak oxygen uptake $\left(\dot{V} \mathrm{O}_{\text {2peak }}\right)[2]$.

Evidence of the association between low CRF and a higher morbidity and mortality from all causes, including cardiovascular disease and cancer, has been consistent and strong [3]. An improvement of CRF by $1 \mathrm{ml} / \mathrm{kg} / \mathrm{min}$ (determined using a maximal bike test) has been shown to reduce the risk for developing overweight or obesity by $10 \%$ in 6 years [4]. In addition to that, improving CRF in childhood and adolescence is associated with a healthier cardiovascular profile in later life [5]. Furthermore, a physically active childhood enhances a physically active lifestyle over a life span [6].

As CRF is an objective reproducible physiological measure reflecting the functional influences of physical activity habits, genetics, and disease status [7], the aim should be to improve CRF early in life. However, currently CRF of 25.4 million people aged 6 to 19 years from 27 countries has declined by $3.6 \%$ per decade from 1985 to 2003 [8]. Even though the WHO recommends that children spend at least $60 \mathrm{~min} /$ day of moderate-tovigorous-intensity physical activity [9], most children spend an average of 8 to $9 \mathrm{~h}$ /day in sedentary behavior which in turn tends to increase by $30 \mathrm{~min} /$ day every year in school-aged children [10]. Consequently, the number of children and adolescents with low CRF gradually increases [11]. It is therefore essential to devise effective exercise modalities fit for the use in very young children to change their general movement pattern towards a higher level of physical activity.

There are two training modalities which have been explored in the literature: moderate-intensity continuous training (MICT) and high-intensity interval training (HIIT). In HIIT, short bursts of high-intensity activity alternate with lower-intensity activity for recovery or rest [12-14]. The intensity for the high-intensive bursts is described as "all-out," "maximal effort," " $\geq 90 \%$ $\mathrm{VO}_{2}$ peak," "85-95\% maximum heart rate (HR)," or " $\geq$ $100 \%$ maximal aerobic speed" [14]. MICT on the other hand consists mainly of aerobic exercise training performed continuously or in intervals [15]. The training modality which shows the most promising effects for improving CRF in children, adolescents, and adults is highintensity interval training (HIIT) $[14,16]$. Possible explanations for the success of HIIT compared to MICT are the differing adaptations induced in the mitochondria in the trained muscles and a higher effect on central adaptation such as maximal stroke volume, cardiac output, and blood volume, all being important components of CRF [17-19]. Maybe even more important than its effects is the smaller time commitment which could increase training adherence [20]. Intermittent exercise also represents children's spontaneous physical activity [21] and may therefore be better received in this age group than MICT.

Since HIIT seems to represent an effective approach to achieving improvements in CRF among healthy children and adolescents, we aimed to evaluate a training modality which could be easily accessed by healthy children and already represents an activity widely recognized in that age group: trampolining. A first approach to measuring the intensity of trampoline jumping in children has involved the measuring of accelerometry (ACC)-derived and muscle electromyography (EMG)based estimates [22]. However, the two measurements provided different results, with ACC describing trampoline jumping as being of vigorous intensity as a consequence of the body being hurled through space [22]. But EMG categorized it as being of light and moderate intensity as it only involves brief bursts of muscle activity [22]. Two studies have investigated the physiological demands of trampolining in adults and concluded that trampolining achieved comparable $\mathrm{VO}_{2}$-values as treadmill running at the same intensity [23, 24]. The conclusion of the authors was that even though the cardiopulmonary parameters were significantly higher during trampolining compared to treadmill running, the fact that the trendline associated with each variable was similar, monitoring the intensity of the session based on heart rate during trampolining as is already the custom with running was justified [24].

The question remains, whether trampoline jumping can be categorized as a vigorous or as a moderateintensity physical activity and whether it could be used as a reasonable training modality for HIIT in children.

\section{Material and Methods}

The study was performed in accordance with the standards of ethics outlined in the Declaration of Helsinki and was approved by the Ethics Committee of the University of Erlangen-Nürnberg, FRG (No. 409_19).

\section{Subjects}

Fifteen healthy children aged between 7 and 10 years agreed to participate in this study. Each child as well as their legal guardians gave written informed consent using a consent form approved by the Ethics Committee of the University of Erlangen-Nuremberg, FRG. All participants were Caucasian, non-obese, and healthy and 
were recruited from local schools. All children performed one outdoor incremental running test and then one trampoline jumping test during which the children were asked to jump at different intensities according to a preset protocol.

\section{Body Composition}

Height and weight were measured using a stadiometer and electronic scale (Seca 704 S, Hamburg, Germany).

\section{Measurement of $\dot{\mathrm{VO}}_{2}$}

The cardiopulmonary exercise test was performed using a mobile testing device (Metamax •, Cortex, Leipzig, Germany). A small, low-dead-space respiratory valve $(88 \mathrm{ml})$ with a pediatric mouthpiece and headgear was used. Gas-exchange was measured continuously during each test using a breath-by-breath method and averaged over 15-s intervals. Criteria for completion of a valid peak $\mathrm{VO}_{2}$ test included two of the following three criteria: (1) $\mathrm{HR} \geq 200$ beats/min $(\mathrm{bpm}),(2)$ respiratory exchange ratio (RER) $\geq 1.0$, and (3) volitional fatigue [25]. All children were instructed to abstain from food or carbohydrate-rich drinks for $2 \mathrm{~h}$ leading up to the test. Ventilatory thresholds $\mathrm{VT}_{1}$ and $\mathrm{VT}_{2}$ were determined using the $\mathrm{V}$-slope method proposed by Wasserman et al. [26].

\section{Outdoor Incremental Running Test Protocol}

The test was performed outdoors using an incremental step test previously described [27]. After a warm-up and a short rest period, each child was equipped with a heart rate monitor (Polar H7 Bluetooth Smart $4.0^{\circ}$ heartrate sensor, Kempele, Finnland) and the mobile exercise equipment. The mask was fitted and the backpack containing the mobile exercise equipment (MetaMax $3 \mathrm{~B}^{\circ}$, Cortex, Leipzig, Germany) adjusted, so it would not move on the child's torso.

The test consisted of 4 steps of 2-min lengths. The first 2 min consisted of walking at a leisurely pace. After that, the children were instructed to increase their speed to an easy jog. After $2 \mathrm{~min}$ at this speed, they were instructed to run with some effort for another $2 \mathrm{~min}$ and then to increase their speed to as fast as possible and maintain this speed for as long as possible. Once they could no longer maintain their maximum speed or were too exerted to keep going, the test was ended. An experienced researcher and running coach for children performed the whole test alongside each child control-

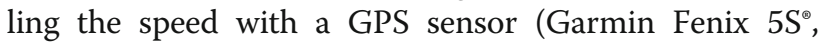
Garmin, Olathe, USA). This was important for slowing the children down during the first two steps and then to motivate them during the last step. Running was performed on a flat and even trail and children were instructed to use sportive footwear and clothing. We did not perform a treadmill test, as this outdoor incremental running test protocol has proven its efficacy this age group [28].

\section{Trampoline Test Protocol}

A minimum of 2 weeks later, all children were tested again on the trampoline. They were first fitted with the mobile cardiopulmonary exercise testing device and the heart rate monitor as described above. To assess the intensity during leisurely jumping as well as during vigorous exercise, the protocol consisted of $5 \mathrm{~min}$ of easy hopping. During these first $5 \mathrm{~min}$, the children were instructed to jump very little and to not overexert themselves. After this phase of moderate intensity, followed a rest period of $1 \mathrm{~min}$ which the children spent standing still. Then, followed 2 min of vigorous jumping, during which the children were motivated to jump as high as possible. After a second rest period of 1 min spent standing still another bout of vigorous exercise was performed for another $2 \mathrm{~min}$. All recorded values were then averaged over the duration of each interval. Additionally, the maximum values reached by each child during the periods of vigorous jumping were recorded.

\section{Statistical Analysis}

Statistical analysis was performed using Microsoft Excel $2000^{\circ}$ for data collection and SPSS $12.0^{\circ}$ (SPSS Inc., Chicago, IL). All measured values are reported as means and standard deviations. The Kolmogorov-Smirnov test was used to check for normal distribution. Homogeneity of variance was investigated using Levine's F-test. For normally distributed variables, differences between the two test protocols were assessed with paired t-tests; otherwise, the Wilcoxon or the Whitney-Mann U-tests were used. All tests were 2-tailed; a 5\% probability level was considered significant (*).

\section{Results}

\section{Subjects}

We were able to test 15 children outdoors and on the trampoline. Out of the 15 children (mean age 8.8 years, mean weight $27.6 \mathrm{~kg}$, height $133.4 \mathrm{~cm}$, body mass index (BMI) $15.3 \mathrm{~kg}^{-2}$ ), 10 were boys (mean age 8.8 years, weight $28.9 \mathrm{~kg}$, height $135.7 \mathrm{~cm}$, BMI $15.5 \mathrm{~kg}^{-2}$ ) and 5 were girls (mean age 8.7 years, weight $25 \mathrm{~kg}$, height $128.8 \mathrm{~cm}$, BMI $15 \mathrm{~kg}^{-2}$ ). The boys and girls did not differ significantly from each other with respect to age or anthropometric variables. When asked about the subjective effort, all children felt tired after having performed the trampoline test but also agreed that they had enjoyed themselves. 


\section{Oxygen Uptake $\left(\mathrm{V}_{2}\right)$}

An example of $\mathrm{VO}_{2}$ over the course of the trampoline test is represented in Fig. 1 along with the $\mathrm{VO}_{2}$-peak value recorded for the outdoor incremental running test for this child. The oxygen uptake values of all the participants are represented in Fig. 2 as well as in Table 1. The mean $\mathrm{VO}_{2}$ achieved during the 5 min of trampoline jumping of moderate intensity was significantly higher than the $\mathrm{VO}_{2}$ at VT1 determined during the outdoor incremental running test. During both intervals of vigorous jumping, comparable values to VT2, determined during the outdoor incremental running test, were achieved. When compared to $\dot{V} O_{2 p e a k}$ from the outdoor incremental running test, the mean values during the first interval of higher intensity were significantly lower but were comparable during the second 2-min interval of higher intensity. The maximum $\mathrm{VO}_{2}$ determined during the trampoline jumping was significantly higher than $\dot{V} O_{2 p e a k}$ determined during the outdoor incremental running test.

\section{Heart Rate}

The heart rate values for the trampoline and the outdoor incremental running test are represented in Table 1. The mean heart rate during the interval of moderate intensity was comparable to the heart rate at VT1 during the outdoor incremental running test. The same was true for the heart rate at VT2 and the HR during the second high-intensity interval which were also comparable. The maximum heart rate achieved during the trampoline test was not significantly higher than the one achieved during the outdoor incremental running test.

\section{Oxygen Pulse $\left(\mathrm{O}_{2}\right.$-pulse) and Minute Ventilation ( $(\dot{V} E)$}

The parameters for $\mathrm{O}_{2}$-pulse and $V E$ are represented in Table 1. The peak $\mathrm{O}_{2}$-pulse was comparable to the $\mathrm{O}_{2}$ pulse during both high-intensity intervals and significantly higher than during the interval of moderate intensity. The maximum $\mathrm{O}_{2}$-pulse recorded during the trampoline test was significantly higher than during the outdoor incremental running test. Mean peak $\dot{V} E$ was significantly higher during the outdoor incremental running test than during the trampoline test even though the maximum recorded value of $\dot{V} E$ during the trampoline test was comparable to peak $\dot{V} E$ during the outdoor incremental running test. Accordingly, the mean $\dot{V} E$ recorded during the interval of moderate intensity was also significantly lower than the peak $\dot{V} E$ from the outdoor incremental running test.

\section{Discussion}

Trampoline jumping is a widely used physical activity in children. Therefore, trampoline jumping represents an ideal means for promoting physical activity in children. In order to evaluate its intensity during different jumping modalities, we investigated slow jumping with moderate intensity over a period of $5 \mathrm{~min}$ and high-intensity jumping over a period of $2 \mathrm{~min}$.

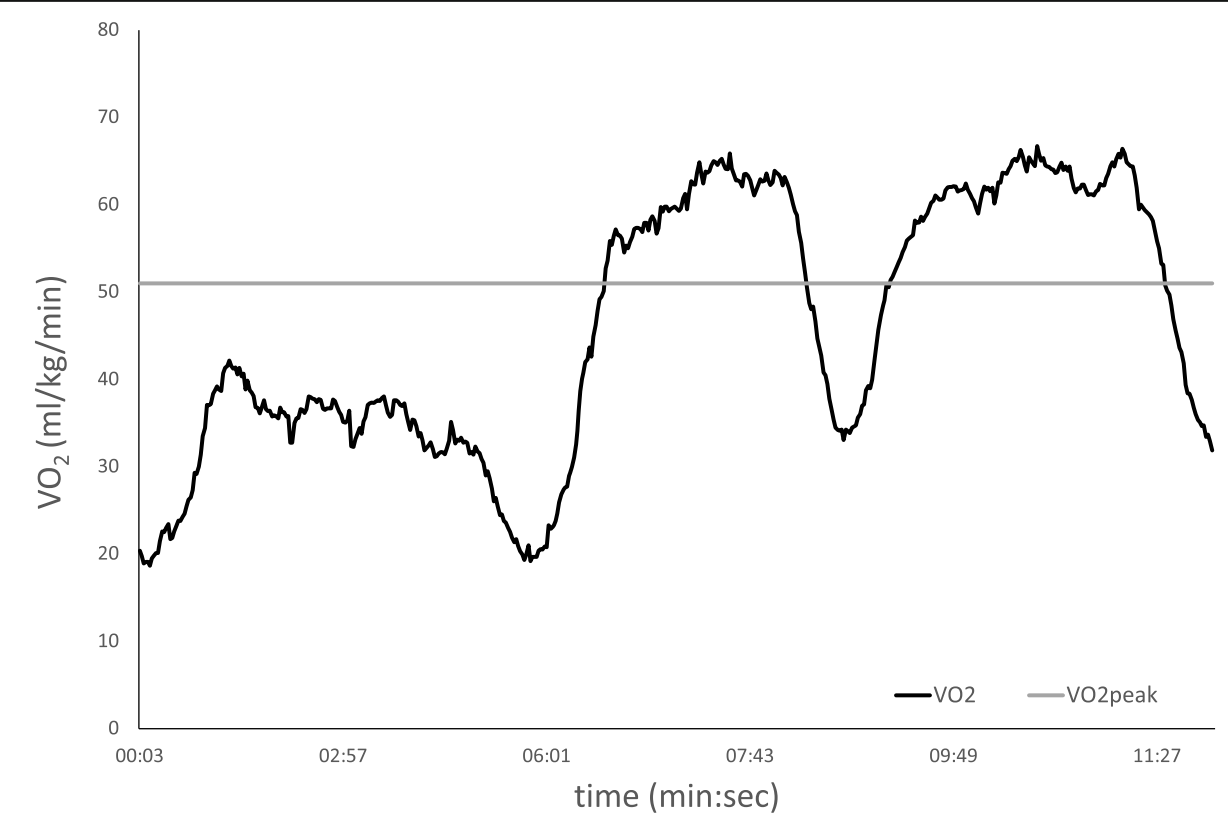

Fig. $1 \mathrm{An}$ example of $\mathbf{V O}_{2}$ over the course of the trampoline test along with the $\mathbf{V O}_{2}$-peak value recorded for the outdoor incremental running test of one child $\left(\mathrm{VO}_{\mathbf{2}}\right.$, oxygen uptake) 


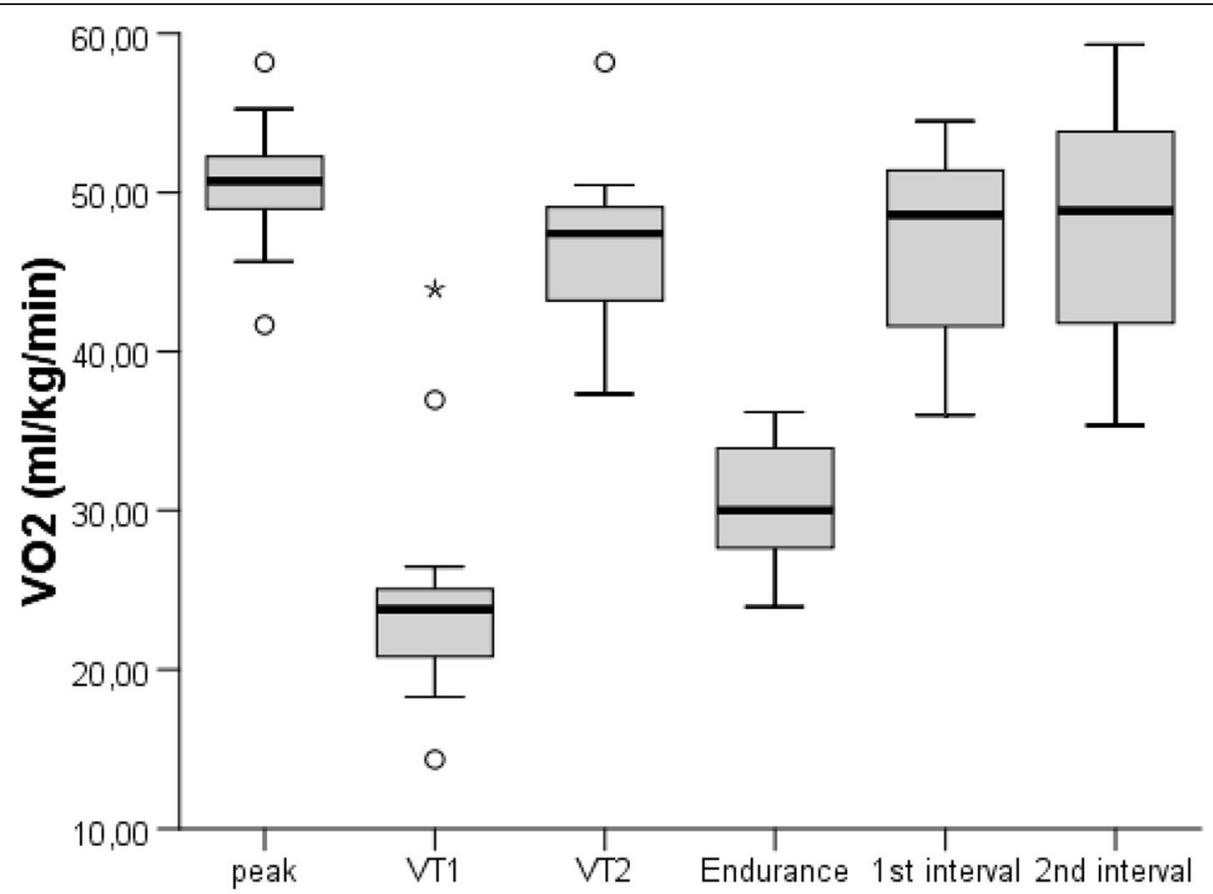

Fig. 2 Median values of the oxygen uptake $\left(\mathbf{V O}_{2}\right)$ at the first $(\mathrm{VT} 1)$ and second (VT2) ventilatory threshold, and the median values recorded for the interval of moderate intensity, the first and second high-intensity intervals as well as the maximum oxygen uptake during the trampoline test, alongside with the values for minimum, maximum, and the 25th and 75th quartile of all participants (degree symbol denotes far outliers (1 interquartile range of 1.5) and asterisk denotes extreme outliers (2 interquartile ranges of 1.5))

\section{Eligibility of Trampoline Jumping for MICT and HIIT}

The first interval of the trampoline test which consisted of slow and mild jumping was supposed to represent a moderate continuous intensity. However, $\dot{V} \mathrm{O}_{2 p e a k}$ during this first interval was already higher than $\dot{V O} O_{2}$ at VT1 from the outdoor incremental running test. Still, the heart rate achieved during this interval of moderate jumping and the HR achieved at VT1 during the outdoor incremental running test was comparable, indicating that the heart rate is not an accurate tool for estimating cardiopulmonary exertion. The same observation was made for $\mathrm{VO}_{2}$ at VT2 during the outdoor incremental running test which was comparable to $\dot{V O}_{2}$ during both high-intensity intervals. However, the mean heart rate was significantly lower during the first high- intensity interval on the trampoline when compared to the VT2 of the outdoor incremental running test. The mean heart rate on the trampoline only rose enough during the second high-intensity interval on the trampoline to reach comparable values to the HR recorded at VT2 during the outdoor incremental running test. Slow trampoline jumping could therefore be an adequate tool for moderate-intensity continuous training in children as it can be performed close to $\mathrm{VO}_{2}$ at VT1. However, the children stated that they were bored during the slow jumping and felt no tiredness afterwards. The vigorous jumping was much more enjoyable according to their accounts and was performed with $\dot{V} \mathrm{O}_{2}$-values comparable to VT2 from the outdoor incremental running test, thus representing high-intensity interval training.

Table 1 Mean values recorded during the incremental running outdoor test compared to the mean values for the three jumping intervals as well as the mean values of the maximum achieved by each child during the trampoline jumping test $\left(\dot{V} \boldsymbol{O}_{\mathbf{2}}\right.$ oxygen uptake, $\mathrm{O}_{2}$-pulse oxygen pulse, $\boldsymbol{V} \boldsymbol{E}$ minute ventilation, VT1 first aerobic threshold, VT2 second ventilatory threshold)

\begin{tabular}{|c|c|c|c|c|c|c|c|}
\hline & \multicolumn{3}{|c|}{ Running test } & \multicolumn{3}{|c|}{ Trampoline test } & \multirow[t]{2}{*}{ Maximum } \\
\hline & Peak & VT1 & VT2 & Endurance & 1st interval & 2nd interval & \\
\hline $\mathrm{VO}_{2}\left(\mathrm{~mL} \mathrm{~kg}{ }^{-1} \mathrm{~min}^{-1}\right)$ & $50.4(4)$ & $24.6(7.3)$ & $46.2(5.4)$ & $30.4(4.1)$ & $47.1(5.6)$ & $48.3(6.9)$ & $55.6(6.7)$ \\
\hline $\mathrm{O}_{2}$-pulse $\left(\mathrm{mL}\right.$ beat $\left.^{-1}\right)$ & $7.4(1.9)$ & & & $6.2(1.1)$ & $7.4(1.6)$ & $7.5(1.8)$ & $8.2(2)$ \\
\hline Heart rate $\left(\min ^{-1}\right)$ & $191.7(8.3)$ & $130.4(12.6)$ & $183(10.3)$ & $133.6(10.8)$ & $173.8(10.2)$ & $183(10.3)$ & $189.6(8.3)$ \\
\hline$\dot{V} E\left(\mathrm{~L} \mathrm{~min}^{-1}\right)$ & $53.9(11.2)$ & & & $24.3(3)$ & $45.6(9.3)$ & $47(11)$ & $52.9(12)$ \\
\hline
\end{tabular}


The mean $\dot{V O}_{2}$ achieved during the second highintensity interval was comparable to $\dot{V} O_{2 p e a k}$ from the incremental step test, and the maximum $\mathrm{VO}_{2}$ recorded during the trampoline test was significantly higher. Additionally, the children were not only able to achieve this high-oxygen uptake during vigorous trampoline jumping but were also able to sustain this high intensity for a duration of $2 \mathrm{~min}$ as can be seen in the exemplary data represented in Fig. 1. These findings suggest that a true $\mathrm{VO}_{2} \max$ can be achieved during trampoline jumping by kids.

Two previous studies have investigated the cardiopulmonary effects of trampolining [23, 24]. The maximum $\mathrm{VO}_{2}$ recorded during vigorous jumping was around $40 \mathrm{ml} \mathrm{kg} \mathrm{min}^{-1}$ in healthy, adult males [23, 24], which is significantly lower than the maximum recorded in our study $\left(55.6 \mathrm{ml} \mathrm{kg}{ }^{-1} \mathrm{~min}^{-1}\right)$. Children tend to achieve higher $\mathrm{VO}_{2}$-values than adults during cardiopulmonary exercise tests [29], which can explain this discrepancy. In these studies, $\mathrm{VO}_{2}$-values during vigorous trampolining did not differ significantly from vigorous running on the treadmill [23, 24], whereas the children in our study achieved significantly higher values on the trampoline than during the outdoor incremental running test. One explanation could be that the children were maybe more willing to fully exhaust themselves on the trampoline than while running outdoors. It could also be that larger muscle groups are recruited during vigorous trampolining than when running. Comparable test protocols are needed to evaluate this finding further.

\section{Cardiac and Pulmonary Contribution During Trampoline Jumping}

The cardiac output, indirectly measured through the $\mathrm{O}_{2}$ pulse, was comparable during both high-intensity intervals and at $\dot{V} O_{2 p e a k}$ during the outdoor incremental running test. Interestingly, the minute ventilation $\dot{V} E$ was significantly lower during both high-intensity intervals than at $\dot{V} O_{2 p e a k}$ during the outdoor incremental running test, but the maximum value during trampoline jumping and incremental step test was comparable. This indicates that peak $\mathrm{O}_{2}$-pulse was reached by the children during the trampoline test and that they were able to maintain this high cardiac output for the full duration of the highintensity interval. On the other hand, even though peak $\dot{V} E$ reached during trampoline jumping and outdoor running was comparable, the mean value for $\dot{V} E$ recorded during the high-intensity interval on the trampoline was lower than peak $\dot{V} E$ recorded during the outdoor incremental running test. This could be due to a slow rise of $\dot{V} E$, reaching maximum values only towards the end of the 2min interval. Since mean $\mathrm{O}_{2}$-pulse and mean $\mathrm{HR}$ achieved during the vigorous trampoline jumping were comparable to peak $\mathrm{O}_{2}$-pulse and peak $\mathrm{HR}$ from the outdoor incremental running test, the significant difference between mean $\dot{V} E$ achieved during the trampoline test and peak $\dot{V} E$ achieved during the outdoor incremental running test underlines the fact that the main limitation during maximum exertion is of a cardiac and not a pulmonary nature. The children reach their peak $\mathrm{O}_{2}$-pulse and heart rate so early in the 2-min interval of vigorous trampolining as to reach mean values that are comparable to peak $\mathrm{O}_{2}$-pulse and $\mathrm{HR}$ recorded during the outdoor incremental running test. However, they only reached the peak $\dot{V} E$ from the outdoor incremental running test at the very end of this 2-min interval of vigorous jumping.

This study has several limitations. First, the number of participants is small and more significant differences might have become apparent with a higher number of children. There was no evaluation of the power realized during the trampoline jumping as the determination of metabolic equivalents for trampoline jumping has not been established yet.

\section{Conclusion}

As the test subjects in this study were able to achieve $\dot{V}$ $\mathrm{O}_{2}$-values at VT2 which they were then able to maintain for a duration of $2 \mathrm{~min}$ twice with only $1 \mathrm{~min}$ rest in between, trampoline jumping could be a very effective way to achieve HIIT in young children. The children all stated that they had tremendously enjoyed the vigorous jumping and more than the slow jumping of moderate intensity. As it is a readily available training device for most families-either at home or in a near-by facility-it could be used for promoting physical activity in children through high-intensity interval training interventions.

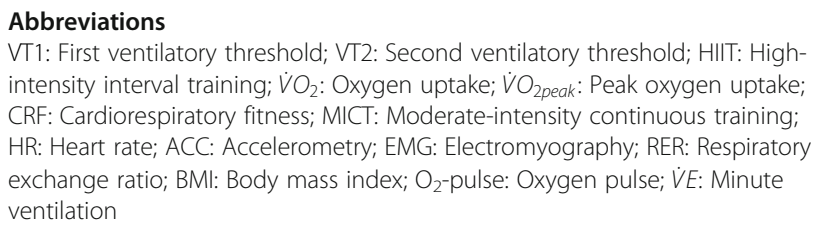
intensity interval training; $\dot{V}_{2}$ : Oxygen uptake; $\dot{V} O_{2 p e a k}$ : Peak oxygen uptake; CRF: Cardiorespiratory fitness; MICT: Moderate-intensity continuous training; HR: Heart rate; ACC: Accelerometry; EMG: Electromyography; RER: Respiratory exchange ratio; $\mathrm{BMI}$ : Body mass index; $\mathrm{O}_{2}$-pulse: Oxygen pulse; $\dot{V} E$ : Minute ventilation

\section{Acknowledgements}

No acknowledgements

\section{Code Availability}

Not applicable

\section{Authors' Contributions}

IS, BE, KR, AW, SD, VS Conception and design of the study. IS, BE, KR, AW, VS Data acquisition. IS, BE, KR, AW Data analysis and interpretation. IS, BE Drafting of the manuscript. IS, BE, KR, AW, SD, VS Critical revision of the manuscript. IS, BE, KR, AW, SD, VS Accountability for all aspects of work, ensuring integrity and accuracy. All authors of the submitted manuscript certify that they have sufficiently participated in the work to take responsibility for the manuscript's content. Furthermore, each author certifies that this material has not been and will not be submitted to or published in any other publication. The authors read and approved the final manuscript.

Funding

Open Access funding enabled and organized by Projekt DEAL. 


\section{Availability of Data and Materials}

Can be made available upon reasonable request

\section{Declarations}

\section{Ethics Approval and Consent to Participate}

The study was approved by the Ethical Board of the University of ErlangenNürnberg, No. 409_19. Each child as well as their legal guardians gave written informed consent using a consent form approved by the Ethics Committee of the University of Erlangen-Nuremberg, FRG.

\section{Consent for Publication}

Not applicable. Patients or the public were not involved in the design, conduct, reporting, or dissemination plans of our research.

\section{Competing Interests}

The authors, Isabelle Schöffl, Benedikt Ehrlich, Kathrin Rottermann, Annika Weigelt, Sven Dittrich, and Volker Schöffl, declare that they have no competing interests.

\section{Author details}

'Department of Pediatric Cardiology, University Hospital Erlangen-Nuremberg, 91054 Erlangen, Germany. ${ }^{2}$ School of Clinical and Applied Sciences, Leeds Beckett University, Leeds, Great Britain. ${ }^{3}$ Section of Sportsmedicine and Sports Orthopaedics, Department of Orthopedic and Trauma Surgery, Klinikum Bamberg, Bamberg, Germany. ${ }^{4}$ Section of Wilderness Medicine, Department of Emergency Medicine, University of Colorado School of Medicine, Denver, USA.

Received: 12 June 2020 Accepted: 8 June 2021

Published online: 30 July 2021

\section{References}

1. Sui X, LaMonte MJ, Blair SN. Cardiorespiratory fitness as a predictor of nonfatal cardiovascular events in asymptomatic women and men. Am J Epidemiol. 2007;165(12):1413-23. https://doi.org/10.1093/aje/kwm031.

2. Ross R, Blair SN, Arena R, Church TS, Despres JP, Franklin BA, et al. Importance of assessing cardiorespiratory fitness in clinical practice: a case for fitness as a clinical vital sign: a scientific statement from the American Heart Association. Circulation. 2016;134(24):e653-e99. https://doi.org/10.11 61/CIR.0000000000000461.

3. Kodama S, Saito K, Tanaka S, Maki M, Yachi Y, Asumi M, et al. Cardiorespiratory fitness as a quantitative predictor of all-cause mortality and cardiovascular events in healthy men and women: a meta-analysis. JAMA. 2009:301(19):2024-35. https://doi.org/10.1001/jama.2009.681.

4. Ortega FB, Lavie CJ, Blair SN. Obesity and cardiovascular disease. Circ Res. 2016:118(11):1752-70. https://doi.org/10.1161/CIRCRESAHA.115.306883.

5. Ruiz JR, Castro-Pinero J, Artero EG, Ortega FB, Sjostrom M, Suni J, et al. Predictive validity of health-related fitness in youth: a systematic review. $\mathrm{Br} J$ Sports Med. 2009;43(12):909-23. https://doi.org/10.1136/bjsm.2008.056499.

6. Telama R, Yang X, Leskinen E, Kankaanpaa A, Hirvensalo M, Tammelin T, et al. Tracking of physical activity from early childhood through youth into adulthood. Med Sci Sports Exerc. 2014;46(5):955-62. https://doi.org/10.1249/ MSS.0000000000000181.

7. Sui X, Laditka JN, Hardin JW, Blair SN. Estimated functional capacity predicts mortality in older adults. J Am Geriatr Soc. 2007;55(12):1940-7. https://doi. org/10.1111/j.1532-5415.2007.01455.x.

8. Tomkinson GR, Olds TS. Secular changes in pediatric aerobic fitness test performance: the global picture. Med Sport Sci. 2007;50:46-66. https://doi. org/10.1159/000101075.

9. Organization WH. Global recommendations on physical activity for health; 2010. p. 17-21. https://doi.org/10.1080/11026480410034349.

10. Tanaka C, Reilly JJ, Huang WY. Longitudinal changes in objectively measured sedentary behaviour and their relationship with adiposity in children and adolescents: systematic review and evidence appraisal. Obes Rev. 2014;15(10):791-803. https://doi.org/10.1111/obr.12195

11. Martinez-Gomez D, Ortega FB, Ruiz JR, Vicente-Rodriguez G, Veiga OL, Widhalm K, et al. Excessive sedentary time and low cardiorespiratory fitness in European adolescents: the HELENA study. Arch Dis Child. 2011:96(3):240U6. https://doi.org/10.1136/adc.2010.187161.
12. Norton K, Norton L, Sadgrove D. Position statement on physical activity and exercise intensity terminology. J Sci Med Sport. 2010;13(5):496-502. https:// doi.org/10.1016/j.jsams.2009.09.008.

13. Sloth M, Sloth D, Overgaard K, Dalgas U. Effects of sprint interval training on VO2max and aerobic exercise performance: a systematic review and metaanalysis. Scand J Med Sci Sports. 2013;23(6):e341-52. https://doi.org/1 $0.1111 /$ sms.12092

14. Cao M, Quan M, Zhuang J. Effect of high-intensity interval training versus moderate-intensity continuous training on cardiorespiratory fitness in children and adolescents: a meta-analysis. Int J Environ Res Public Health. 2019:16(9):1533. https://doi.org/10.3390/ijerph16091533.

15. Araujo BTS, Leite JC, Fuzari HKB, Pereira de Souza RJ, Remigio MI, Dornelas de Andrade $A$, et al. Influence of high-intensity interval training versus continuous training on functional capacity in individuals with heart failure: a systematic review and meta-analysis. J Cardiopulm Rehabil Prev. 2019;39(5): 293-8. https://doi.org/10.1097/HCR.0000000000000424.

16. Cao M, Quan M, Zhuang J. Effect of high-intensity interval training versus moderate-intensity continuous training on cardiorespiratory fitness in children and adolescents: a meta-analysis. Int J Environ Res Public Health. 2019;16(9). https://doi.org/10.3390/ijerph16091533.

17. Astorino TA, Allen RP, Roberson DW, Jurancich M. Effect of high-intensity interval training on cardiovascular function, VO2max, and muscular force. J Strength Cond Res. 2012;26(1):138-45. https://doi.org/10.1519/JSC.0b013e31 $8218 \mathrm{dd} 77$.

18. Helgerud J, Hoydal K, Wang E, Karlsen T, Berg P, Bjerkaas M, et al. Aerobic highintensity intervals improve VO2max more than moderate training. Med Sci Sports Exerc. 2007;39(4):665-71. https://doi.org/10.1249/mss.0b013e3180304570.

19. Daussin FN, Zoll J, Ponsot E, Dufour SP, Doutreleau S, Lonsdorfer E, et al. Training at high exercise intensity promotes qualitative adaptations of mitochondrial function in human skeletal muscle. J Appl Physiol (1985). 2008:104(5):1436-41. https://doi.org/10.1152/japplphysiol.01135.2007.

20. Batacan RB Jr, Duncan MJ, Dalbo VJ, Tucker PS, Fenning AS. Effects of highintensity interval training on cardiometabolic health: a systematic review and meta-analysis of intervention studies. Br J Sports Med. 2017:51(6):494503. https://doi.org/10.1136/bjsports-2015-095841.

21. Baquet G, Dupont G, Gamelin FX, Aucouturier J, Berthoin S. Active versus passive recovery in high-intensity intermittent exercises in children: an exploratory study. Pediatr Exerc Sci. 2019;31(2):248-53. https://doi.org/1 0.1123/pes.2018-0218

22. Gao Y, Melin M, Mäkäräinen K, Rantalainen T, Pesola AJ, Laukkanen A, et al. Children's physical activity and sedentary time compared using assessments of accelerometry counts and muscle activity level. PeerJ. 2018;6:e5437. https://doi.org/10.7717/peerj.5437.

23. Bhattacharya A, McCutcheon EP, Shvartz E, Greenleaf JE. Body acceleration distribution and $\mathrm{O} 2$ uptake in humans during running and jumping. J Appl Physiol Respir Environ Exerc Physiol. 1980;49(5):881-7. https://doi.org/10.11 52/jappl.1980.49.5.881.

24. Draper N, Clement T, Alexander K. Physiological demands of trampolining at different intensities. Res Q Exerc Sport. 2019:1-6. https://doi.org/10.1080/02 701367.2019.1651448

25. McManus A, Leung M. Maximising the clinical use of exercise gaseous exchange testing in children with repaired cyanotic congenital heart defects: the development of an appropriate test strategy. Sports Med. 2000; 29(4):229-44. https://doi.org/10.2165/00007256-200029040-00002.

26. Beaver WL, Wasserman K, Whipp BJ. A new method for detecting anaerobic threshold by gas exchange. J Appl Physiol. 1986;60(6):2020-7. https://doi. org/10.1152/jappl.1986.60.6.2020.

27. Schöffl I EB, Stanger S, Rottermann K, Dittrich S, Schöffl V. A new protocol for cardiopulmonary exercise field testing in children pediatric cardiology. 2020. under review.

28. Schoffl I, Ehrlich B, Stanger S, Rottermann K, Dittrich S, Schoffl V. Exercise field testing in children: a new approach for age-appropriate evaluation of cardiopulmonary function. Pediatr Cardiol. 2020. https://doi.org/10.1007/ s00246-020-02359-2.

29. Takken T, Verschuren O. Commentary on "development of reference values for the functional mobility assessment". Pediatr Phys Ther. 2012;24(3):230-1. https://doi.org/10.1097/PEP.0b013e31825c8b62.

\section{Publisher's Note}

Springer Nature remains neutral with regard to jurisdictional claims in published maps and institutional affiliations. 